\title{
Preventing Fusarium Head Blight of Wheat and Cob Rot of Maize by Inhibition of Fungal Deoxyhypusine Synthase
}

\author{
Mayada Woriedh, ${ }^{1}$ Ilona Hauber, ${ }^{2}$ Ana Lilia Martinez-Rocha, ${ }^{1}$ Christian Voigt, ${ }^{1}$ Frank J. Maier, ${ }^{1}$ \\ Marcus Schröder, ${ }^{3}$ Chris Meier, ${ }^{3}$ Joachim Hauber, ${ }^{2}$ and Wilhelm Schäfer ${ }^{1}$ \\ ${ }^{1}$ Department of Molecular Phytopathology and Genetics, Biocenter Klein Flottbek, University of Hamburg, Ohnhorststrasse 18, \\ D-22609 Hamburg, Germany; ${ }^{2}$ Heinrich-Pette-Institute for Experimental Virology and Immunology, Martinistrasse 52, \\ D-20251 Hamburg, Germany; ${ }^{3}$ Department of Chemistry, Organic Chemistry, University of Hamburg, Martin-Luther-King- \\ Platz 6, D-20146 Hamburg, Germany
}

Submitted 21 March 2010. Accepted 20 December 2010.

Upon posttranslational activation, the eukaryotic initiation factor-5A (eIF-5A) transports a subset of mRNAs out of the nucleus to the ribosomes for translation. Activation of the protein is an evolutionary highly conserved process that is unique to eIF-5A, the conversion of a lysine to a hypusine. Instrumental for the synthesis of hypusine is the first of two enzymatic reactions mediated by deoxyhypusine synthase (DHS). We show that DHS of wheat and the pathogenic fungus Fusarium graminearum, which causes one of the most destructive crop diseases worldwide, are transcriptionally upregulated during their pathogenic interaction. Although DHS of wheat, fungus, and human can be equally inhibited by the inhibitor CNI-1493 in vitro, application during infection of wheat and maize flowers results in strong inhibition of the pathogen without interference with kernel development. Our studies provide a novel strategy to selectively inhibit fungal growth without affecting plant growth. We identified fungal DHS as a target for the development of new inhibitors, for which CNI-1493 may serve as a lead substance.

Polyamines are required for normal growth of microorganisms, including fungi (Foster and Walters 1993; Havis et al. 1994). Spermidine is known to be the predominant polyamine in fungi. The inhibition of spermidine synthase proved to be effective in reducing fungal growth in culture (Stevens and Winther 1979). Thus, spermidine analogs were shown to possess antifungal and fungicidal activity, in spite of the fact that spermidine inhibitors did not exert their antifungal effects via disruption of other cellular functions associated with polyamines (Jakus et al. 1993; Mackintosh and Walters 1997a and b, 2003). As the aminobutyl side chain of hypusine is derived

Corresponding author: Wilhelm Schäfer; Telephone: +49 40 42816266; Fax: +49 40 42816513; E-mail: schaefer@botanik.uni-hamburg.de

Nucleotide sequence data is available in the GenBank database under the following accession numbers: $F$. graminearum FG00323.1, number 2782546 FG00323.1, and Triticum aestivum deoxyhypusine synthase, number FJ376389.1.

* The $e$-Xtra logo stands for "electronic extra" and indicates that four supplementary figures are published online.

This article is in the public domain and not copyrightable. It may be freely reprinted with customary crediting of the source. The American Phytopathological Society, 2011. from spermidine (Park 2006; Wolff et al. 2007), spermidine inhibitors inhibit the biosynthesis of hypusine.

The only hypusine-containing protein identified so far is the eukaryotic initiation factor 5A (eIF-5A). Hypusine is formed in a novel posttranslational modification that involves two enzymes, deoxyhypusine synthase (DHS) and deoxyhypusine hydroxylase (DOHH). DHS (EC 2.5.1.46) catalyzes the cleavage of the polyamine spermidine and transfers its 4-aminobutyl moiety to the $\varepsilon$-amino group of one specific lysine residue of the eIF-5A precursor to form a deoxyhypusine intermediate. In the next step, DOHH (EC 1.14.99.29) converts the deoxyhypusine-containing intermediate to the hypusine-containing mature eIF-5A. Formation of hypusine results in activation of eIF-5A (Park 2006; Wolff et al. 2007).

EIF-5A is a highly conserved protein from archaebacteria to mammals. Hypusine has been described so far in the filamentous fungus Neurospora crassa and in the slime mold Dictyostelium discoideum (Mackintosh and Walters 2003).

Earlier molecular and biochemical studies showed that hypusine-containing eIF-5A is a nucleocytoplasmatic shuttle protein of a subset of mRNAs related to the G1/S cell-cycle transition and unspliced viral mRNAs (Hanauske-Abel et al. 1995; Ruhl et al. 1993). Lethality of gene disruption of DHS in the yeast Saccharomyces cerevisiae (Sasaki et al. 1996; Schnier et al. 1991) proved the essentiality of this factor for cell viability and the proliferation efficiency (Dias et al. 2008; Park et al. 1993a and b). Further studies demonstrated that eIF-5A is involved in vesicular trafficking and cell-wall integrity (Zanelli and Valentini 2007). Recent studies showed that eIF-5A also promotes translation elongation and is required for protein synthesis in yeast (Cano et al. 2008; Frigieri et al. 2008; Lee et al. 2009).

In plants like Arabidopsis thaliana and tomato, Solanum lycopersicum, constitutive suppression of DHS in relation to the amount of residual DHS protein led to pleiotropic phenotypes, e.g., dwarfed growth. Interestingly, these plants also display delayed senescence and an enhanced tolerance to lethal and chronic sublethal environmental stresses (Duguay et al. 2007; Wang et al. 2003, 2005).

The inhibition of DHS by inhibitors results in suppressed growth of actively dividing mammalian cells and demonstrates an antiproliferative potential (Park et al. 1994; Wolff et al. 2007). Screening assays of DHS inhibitors identified the guanylhydrazone CNI-1493 as a novel inhibitor of DHS. It has a potential to suppress replication of human immunodeficiency virus type 1 (HIV-1), which exploits activated eIF-5A during the formation of progeny viruses (Hauber et al. 2005; Sommer 
et al. 2004). Recently, the guanylhydrazone CNI-1493 is considered a promising drug for antimalarial therapy because of its dual combined action of inhibition host-cell proinflammatory cytokine release and parasitic DHS (Specht et al. 2008).

In the present study, we investigated the pathogenic fungus Fusarium graminearum and its interactions with host plants. $F$. graminearum is a necrotrophic, filamentous ascomycete, which causes Fusarium head blight (FHB) and cob rot of maize and is one of the most destructive crop pathogens worldwide. $F$. graminearum causes quantitative losses by reduction of yield and quality losses by contaminating the remaining harvest with mycotoxins. Therefore, fusarioses of cereals pose a major threat to food supply worldwide. We demonstrate the transcriptional activation of $D H S$ of wheat and fungus during their pathogenic interaction. Furthermore, application of the guanylhydrazone CNI-1493 during infection of wheat and maize flowers results in strong inhibition of the pathogen without interference with kernel development. CNI-1493 inhibits fungal DHS and, thereby, prevents fungal germination, and consequently, fungal virulence without disturbing plant growth.

\section{RESULTS}

Isolation of the cDNA sequence of wheat $D H S$.

Comparison of DHS cDNAs from GenBank of rice (Oryza sativa) and Arabidopsis thaliana identified conserved motifs. Previous studies reported the upregulation of $D H S$ in leaves in response to senescence or stress-induced premature senescence (Wang et al. 2001, 2003). Therefore, we isolated mRNA from dark stressed wheat leaves and, using polymerase chain reaction (PCR) primers derived from the conserved DHS cDNA fragments, amplified a 738-bp motif (compare experimental procedures). The PCR fragment of wheat was cloned and sequenced and displayed a homology of $72 \%$ to A. thaliana, $91 \%$ to rice, and $98 \%$ to the motifs. This fragment was then used for designing $5^{\prime}$ - and $3^{\prime}$-end rapid amplification of cDNA ends primers. The obtained full-length cDNA sequence of wheat $D H S$ contains 1,477 base pairs. It includes a $39-b p 5^{\prime}$ noncoding sequence, a 307-bp $3^{\prime}$ noncoding sequence, and an open reading frame coding for a protein of 376 amino acids with a predicted molecular mass of $41.4 \mathrm{kDa}$, (Supplementary Fig. S1).

$D H S$ cDNAs have been cloned from several plant species, including tomato, Arabidopsis, canola, tobacco, and rice (Duguay et al. 2007; Wang et al. 2001, 2003). All share a high sequence similarity to the identified $D H S$ of wheat.

BLAST searches performed in the GenBank expressed sequence tag database revealed that wheat $D H S$ shares the highest

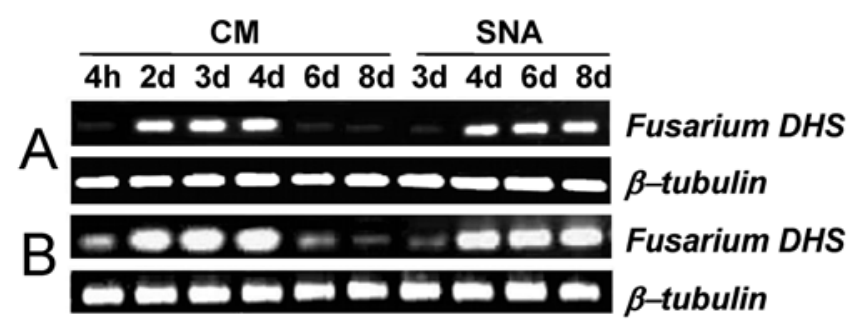

Fig. 1. Expression analysis of the Fusarium graminearum deoxyhypusine synthase (DHS) gene in germinating conidia by reverse transcription-polymerase chain reaction (RT-PCR). RNA isolated from fungal mycelia grown on complete medium after $4 \mathrm{~h}, 2,3,4,6$, and 8 days or synthetic nutrient agar medium after $3,4,6$, and 8 days was used for RT-PCR amplification with $F$. graminearum $D H S$-specific intron spanning primers and $F$. graminearum $\beta$-tubulin-specific intron spanning primers as expression control. Before RT-PCR, RNA samples were digested with DNase I (Qiagen, Hilden, Germany) to remove the contaminating DNA during RNA purification. Semiquantitative RT-PCR was conducted with A, 25 cycles for all samples and $\mathbf{B}, 28$ cycles. similarity to DHS in rice (Oryza sativa). Furthermore, comparison of amino-acid sequences among different species, including Fusarium graminearum FG00323.1, Homo sapiens P49366, Arabidopsis thaliana NM_001036762 and NM_120674, and Oryza sativa NM_001057753 show considerable conservation of sequence identity, particularly in the C-terminal active site of the enzyme, which harbors an enzyme intermediate formation site (Duguay et al. 2007; Wolff and Park 1999). A DHS protein alignment performed with the Clustal W multiple-sequence alignment program (Thompson et al. 1994) is shown in Supplementary Figure S2.

Identification of the DHS gene from Fusarium graminearum. The DHS gene from $F$. graminearum FG00323.1 was identified using a BlastN search against the $F$. graminearum genome database (Munich Information Center for Protein Sequences and Broad Institute) and was published in GenBank with a translated sequence of 358 aa and $1,157 \mathrm{bp}$.

\section{Wheat $\mathrm{DHS}$ and $\mathrm{F}$. graminearum $\mathrm{DHS}$ genes are induced in infected spikes.}

F. graminearum DHS gene induction was analyzed in vitro by isolating RNA from germinating conidia. Interestingly, $\mathrm{DHS}$ gene transcription was detectable in germinating conidia $4 \mathrm{~h}$ after the conidia were grown on complete media. Furthermore, after 2, 3, and 4 days, Fusarium DHS appeared to be strongly induced, while the transcript was reduced remarkably in older mycelium after 6 and 8 days. On poor medium like synthetic nutrient agar (SNA), the transcript of DHS was highly induced after 6 and 8 days (Fig. 1), suggesting a requirement of DHS in germination and fungal growth under stress conditions.

In order to determine whether $D H S$ genes from wheat or Fusarium are transcribed during infection, we inoculated wheat

A infected spikes uninfected wheat plant Control 1dpi 3dpi 7dpi leaf spike stem root

$\begin{array}{ll} & \text { Wheat DHS } \\ 18 S \text { RNA } \\ \text { Fusarium DHS } \\ \text { B-tubulin }\end{array}$

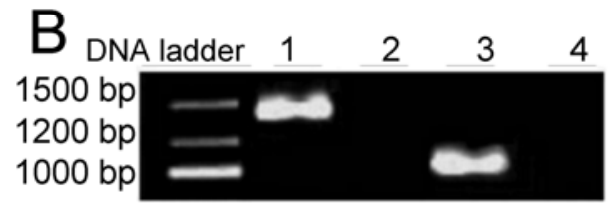

Fig. 2. Expression analysis of wheat and Fusarium graminearum DHS (deoxyhypusine synthase gene) during infection of wheat spikes and noninfected wheat plants by semiquantitative reverse transcription-polymerase chain reaction (RT-PCR). A, RNA was isolated from wheat spikes infected with $F$. graminearum and noninfected spikes as well as from noninfected wheat plant organs such as leaves, stems, roots, and spikes. RT-PCR amplification was performed with $F$. graminearum DHS-specific intron spanning primers or wheat DHS specific primers. Fungal $\beta$-tubulin intron spanning primers or $18 \mathrm{~S}$ rRNA wheat-specific intron spanning primers were used as expression control. Semiquantitative RT-PCR was conducted with 28 cycles for all samples. Before RT-PCR, RNA samples were digested with DNase I (Qiagen, Hilden, Germany) to remove the contaminating DNA during RNA purification. B, Specificity of DHS primers in wheat and $F$. graminearum. cDNA generated from dark stressed wheat leaves was used as template to amplify DHS wheat with wheat DHS primers (lane 1) and with Fusarium DHS primers (lane 2). cDNA generated from germinating conidia was used as template to amplify Fusarium DHS with Fusarium DHS primers (lane 3) and with wheat DHS primers (lane 4). Marker $=$ DNA ladder mix (Fermentas, St. Leon-Rot Germany). 
spikes at the stage of anthesis with $F$. graminearum wild-type conidia. The analysis of uninfected wheat spikes served as control in these experiments.

We analyzed expression patterns using RNA from spikes at 1,3 , and 7 days postinoculation (dpi), comparing them to levels of transcript in organs of uninfected plants. Transcripts of wheat $D H S$ are detectable 3 days postinfection. mRNA levels increased strongly at day 7 postinfection. Fusarium DHS gene transcripts were already detectable after 1 day postinfection and apparently increased during the course of colonization. In sharp contrast, the wheat $D H S$ gene was not expressed in uninfected plants during normal spike development (Fig. 2A). Taken together, these data demonstrated that the DHS gene is differentially transcribed in wheat as well as in Fusarium during infection.

To demonstrate the specificity of the primers, we amplified cDNA generated from dark stressed wheat leaves with Fusa-

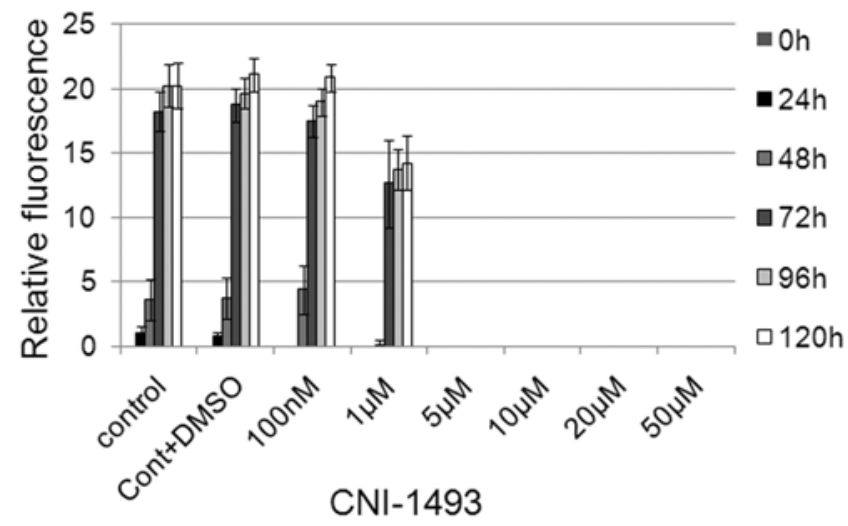

Fig. 3. Inhibition of Fusarium graminearum germination in culture. Conidia suspension $\left(20 \mu \mathrm{l}\right.$, concentration of $2 \times 10^{4}$ conidia per milliliter $)$ of WT-gfp strain and $180 \mu \mathrm{l}$ of synthetic nutrient agar were supplemented with $2 \mu \mathrm{l}$ of CNI-1493 dissolved in dimethyl sulfoxide (DMSO), reaching different concentrations up to $50 \mu \mathrm{M}$ CNI-1493 as indicated and $2 \mu 1$ of pure DMSO as a control. Fluorescence of the wells was determined twice with eight replicates, each at $485 \mathrm{~nm}$ excitation and $538 \mathrm{~nm}$ emissions.

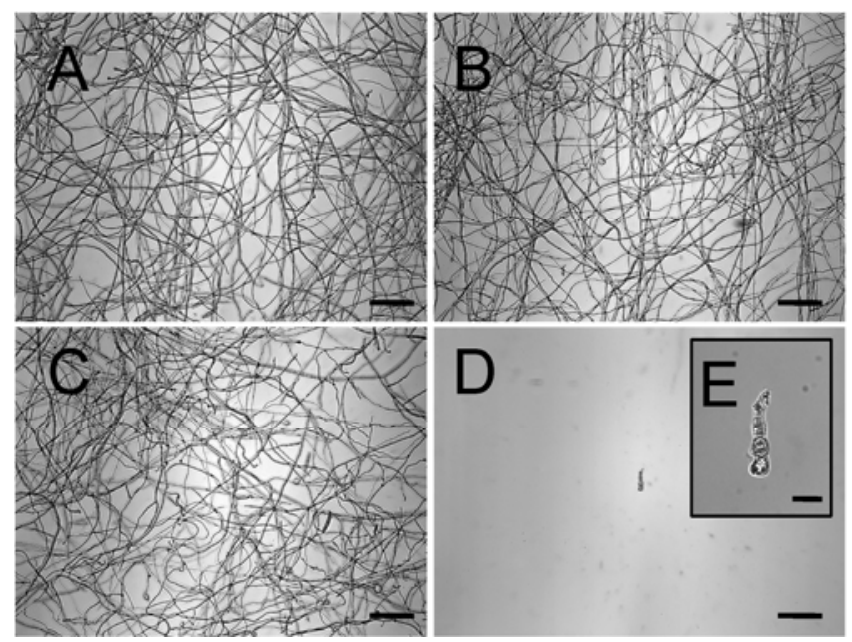

Fig. 4. Inhibition of Fusarium graminearum germination and growth by CNI-1493. F. graminearum conidia were incubated in synthetic nutrient agar medium at $28^{\circ} \mathrm{C}$ and $150 \mathrm{rpm}$ shaking. A, Control, B, dimethyl sulfoxide (DMSO) control (10\% DMSO (vol/vol)), C, CNI-1493 concentration of $100 \mathrm{nM}$ (dissolved in DMSO), D, CNI-1493 concentration of 10 $\mu \mathrm{M}$, and $\mathbf{E}$, magnification of nongerminated conidium from D. Pictures are representative of growth comparing four replicates of each concentration. Pictures were taken by light microscopy after 3 days of incubation. Independent biological samples gave similar results. Scale bars $=50$ (A to D) and $10 \mu \mathrm{m}(\mathrm{E})$. rium primers and cDNA generated from conidia with wheat primers. Both amplifications failed, in contrast to the respective controls (Fig. 2B).

\section{Guanylhydrazone CNI-1493 inhibits germination of $F$. graminearum conidia.}

To test whether CNI-1493 inhibits Fusarium germination in vitro, we inoculated fungal conidia with increasing concentrations of DHS inhibitor. To facilitate quantification of fungal biomass, we used a $F$. graminearum $g f p$-like wild-type strain, WT- $g f p$, that constitutively expresses the gene for the green fluorescent protein (GFP) (Jansen et al. 2005). Conidia of the WT- $g f p$ strain $(n=400)$ were inoculated in SNA medium supplemented with $0,100 \mathrm{nM}, 1,5,10,20$, and $50 \mu \mathrm{M}$ of CNI1493. Spectrofluorometric assays were performed after 24,48 , 72,96 , and $120 \mathrm{~h}$. The subsequent measurement of the relative fluorescence of the constitutively expressed GFP demonstrated a dose-dependent inhibition of germination. Remarkably, CNI1493 completely inhibited Fusarium germination at a concentration of $10 \mu \mathrm{M}$ (Fig. 3). Furthermore, after 3 days of incubation in $10 \mu \mathrm{M}$ CNI-1493, all conidia showed a severely malformed appearance. Approximately $80 \%$ did not germinate at all, while the rest stopped growing after a short germination hypha became visible (Fig. 4).

CNI-1493 concentrations up to $100 \mathrm{nM}$ resulted only in minor fungal growth differences in the microtiter plate cultures, independent of the added chemicals. In contrast, at a concentration of $1 \mu \mathrm{M}$ CNI-1493, a reduction in germination and fungal growth occurred. A complete inhibition of conidia germination was observed at $10 \mu \mathrm{M}$ CNI-1493.

To determine the effect of CNI-1493 on germinated conidia, we inoculated 400 conidia of WT- $g f p$ strain in SNA medium following the addition of final concentrations of 5,10,20, and $100 \mu \mathrm{M}$ CNI-1493 in four different stages: i) fresh conidia $(0 \mathrm{~h}$ postinoculation [hpi]), ii) activated conidia (3 hpi), iii) germinated conidia (6 hpi), or iv) developed hyphae (24 hpi). We performed spectrofluorometric measurements after 24, 48, 72, 97, and 144 h. Results showed that CNI-1493 inhibits germination of $F$. graminearum at 0 and 3 hpi at all tested concentrations. When $5 \mu \mathrm{M}$ of inhibitor was added at 6 and $24 \mathrm{hpi}$, a

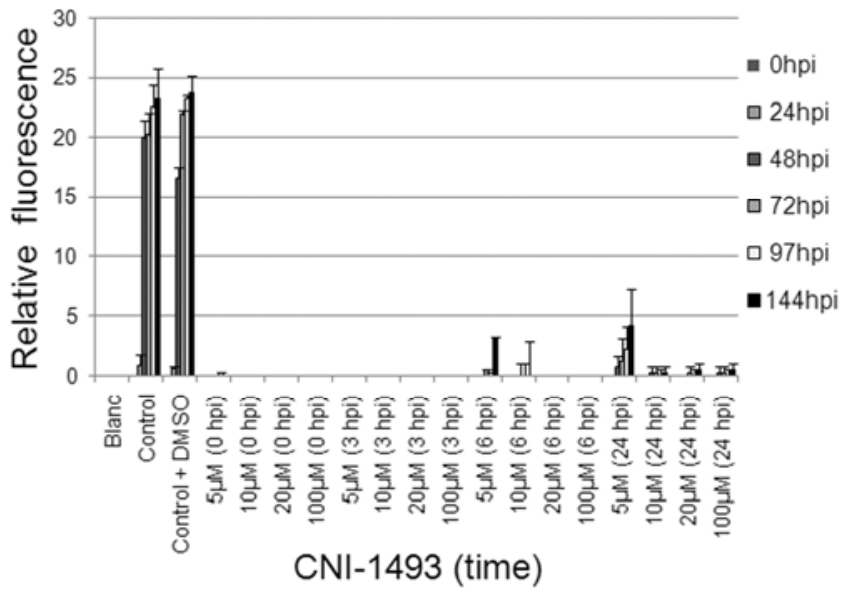

Fig. 5. CNI-1493 inhibits Fusarium graminearum germinated conidia in culture. F. graminearum WT-gfp strain conidia were incubated in synthetic nutrient agar (SNA) media at $28^{\circ} \mathrm{C}$ and $150 \mathrm{rpm}$ shaking. Conidia suspension $(20 \mu \mathrm{l})$ (concentration of $2 \times 10^{4}$ conidia per milliliter) and $180 \mu \mathrm{l}$ of SNA medium were supplemented with $2 \mu \mathrm{l}$ of CNI-1493 dissolved in dimethyl sulfoxide (DMSO), at indicated timepoints and final concentrations. WT- $g f p$ without supplement and WT- $g f p$ plus $2 \mu 1$ DMSO were used as controls. Fluorescence of the wells was determined twice with four replicates each with a fluorometer at $485 \mathrm{~nm}$ excitation and $538 \mathrm{~nm}$ emissions. 
slight increase in fluorescence was observed after $144 \mathrm{~h}$ incubation. Nevertheless, the fluorescence was fivefold less than the control. In addition, when concentrations of $10 \mu \mathrm{M}$ CNI1493 or greater were added at later stages, fluorescence did not increase, indicating $F$. graminearum WT-gfp growth was completely inhibited (Fig. 5). Germinated conidia showed totally impaired growth after $144 \mathrm{~h}$ of incubation with 5, 10, 20, and $100 \mu \mathrm{M}$ CNI-1493 added at 3 hpi (Supplementary Fig. S3). We used this timepoint in further experiments. The analysis of the effect of CNI-1493 on germinated conidia showed a doseand time-dependent inhibition.

\section{The guanylhydrazone CNI-1493 completely suppresses} fungal infection but allows normal kernel development.

To analyze the effect of CNI-1493 on Fusarium infection, we infected wheat spikes and corn cobs with a fungal conidia suspension and added the compound in various concentrations to this suspension. Plants inoculated with conidia from $F$. graminearum wild type and CNI-1493 had drastically suppressed symptoms due to CNI-1493 treatment, as compared with wildtype infected spikes in the absence of this inhibitor. Obviously, a $10-\mu \mathrm{M}$ CNI-1493 concentration prevented fungal infections completely (Fig. 6). On the contrary, kernel development of wheat spikes as well as corn cobs was unaffected. Importantly, these kernels did not differ from water-inoculated control plants. Even the kernels developing from the Fusarium-inoculated wheat spikelets grew normally when the inhibitor was added to

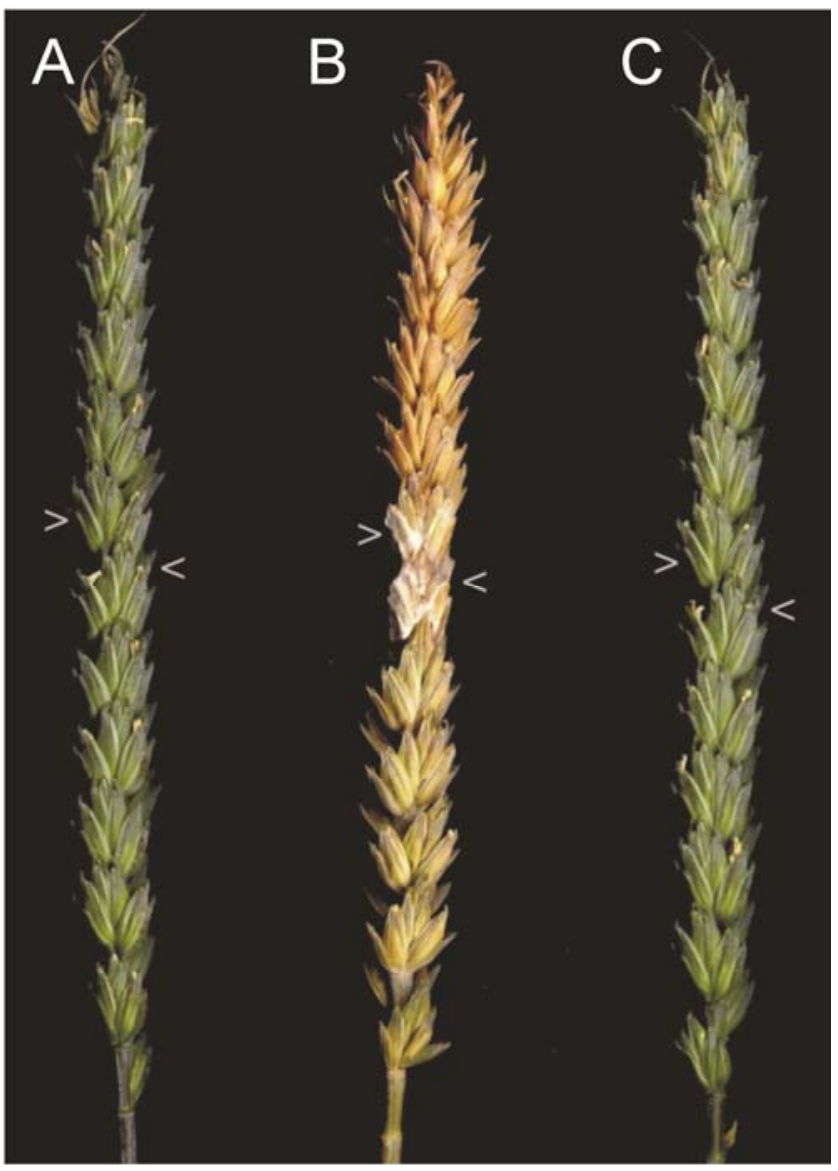

Fig. 6. CNI-1493 inhibits Fusarium graminearum infection of wheat. Picture was taken 21 days postinfection. Seven repetitions were done independently. Arrows indicate inoculation site. Each of two central spikelets were inoculated with $\mathbf{A}, 10 \mu \mathrm{l}$ of water as control, $\mathbf{B}$, wild-type conidial suspension $(10 \mu \mathrm{l})$ (concentration of $2 \times 10^{4}$ conidia per milliliter), $\mathbf{C}$, wild-type conidial suspension $(10 \mu \mathrm{l})$ (concentration of $2 \times 10^{4}$ conidia per milliliter). the inoculums. Inhibitor concentrations lower than $10 \mu \mathrm{M}$ resulted in gradually increasing disease symptoms in maize (Fig. 7). Hence, CNI-1493 suppresses symptom development of $F$. graminearum infections on wheat and maize in a dose-dependent manner. Furthermore, no differences in kernel development were detectable when comparing mock-inoculated to Fusarium inhibitor-inoculated wheat or maize plants. To determine whether the inoculation of wheat kernels with CNI-1493 affects kernel germination or seedling growth, wheat kernels were soaked in different CNI-1493 concentrations of up to $50 \mu \mathrm{M}$ before cultivation. Treatment of wheat kernels with CNI-1493 did not influence their germination or subsequent seedling development (data not shown).

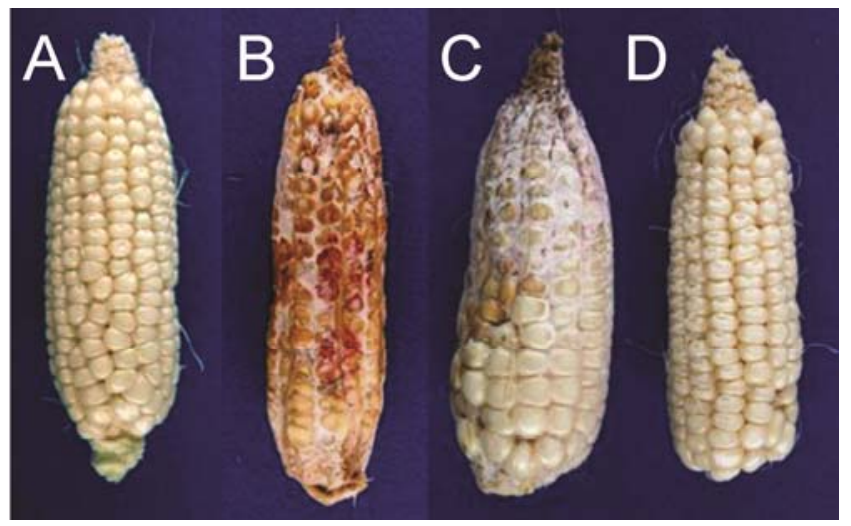

Fig. 7. CNI-1493 inhibits Fusarium graminearum infection of maize cobs Pictures were taken 4 weeks postinfection. Pathogenicity tests were started 3 days postpollination. Eight repetitions were done independently. Cobs were inoculated with $\mathbf{A}, 4 \mathrm{ml}$ of water as control, $\mathbf{B}, 4 \mathrm{ml}$ of $16 \times 10^{4}$ wildtype conidia, C, $4 \mathrm{ml}$ of $16 \times 10^{4}$ wild-type conidia plus $5 \mu \mathrm{M}$ CNI-1493, D, $4 \mathrm{ml}$ of $16 \times 10^{4}$ wild-type conidia plus $10 \mu \mathrm{M} \mathrm{CNI}-1493$.

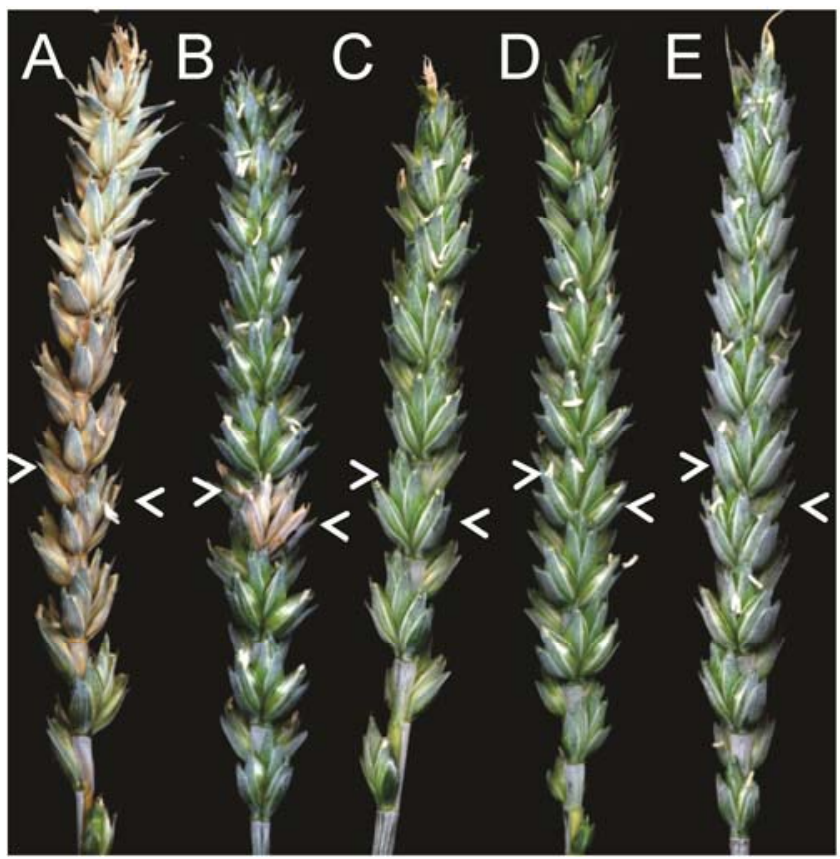

Fig. 8. CNI-1493 inhibits Fusarium graminearum colonization of wheat Pictures were taken 21 days postinoculation, ten repetitions were done independently. Arrows indicate inoculation site. Each of two central spikelets were inoculated with $\mathbf{A}$, wild-type conidial suspension $(10 \mu \mathrm{l})$ (concentration of $2 \times 10^{4}$ conidia per milliliter). B, wild-type conidial suspension $(10 \mu \mathrm{l})$ (concentration of $2 \times 10^{4}$ conidia per milliliter) and $10 \mu \mathrm{l}$ of $200 \mu \mathrm{M}$ CNI-1493 were added $3 \mathrm{~h}$ postinoculation. C, $10 \mu \mathrm{l}$ of $10 \%$ dimethyl sulfoxide as a control and D, 10- $\mu$ l of water as a control. 
To test the effect of separating inhibitor and inoculum during $F$. graminearum infection on wheat, we inoculated wheat spikelets with a conidia suspension of wild type. We added 10 $\mu \mathrm{l}$ of $200 \mu \mathrm{M}$ CNI-1493 to each spikelet 3 hpi. Spikelets inoculated with conidial suspension without the inhibitor, dimethyl sulfoxide (DMSO), or $200 \mu \mathrm{M}$ CNI-1493 were used as controls. We analyzed the infection progress $21 \mathrm{dpi}$. Control spikelets inoculated with CNI-1493 or DMSO did not differ from water-inoculated control plants. In spikelets inoculated with wild type without the inhibitor, the infection of the spike was complete. In contrast, in spikelets inoculated with wild type and the inhibitor, the infection was restricted to the inoculated spikelet (Fig. 8). This indicates that the infection progression is stopped at early stages.

Although the aim of this study is not to establish CNI-1493 as a fungicide, we compared the efficacy of tebuconazole, a known fungicide used against FHB in the field (Homdork et al. 2000; Paul et al. 2007; 2008), and CNI-1493 on wheat spikes infected with $F$. graminearum. We used spray inoculation as a more natural infection method (discussed below). In order to obtain a reproducible infection during spray inoculation, we drastically increased the amount of inoculum. We measured the infection progress 7,14 , and 21 days after inoculation. In wheat spikes sprayed with wild type and $2 \mathrm{mM}$ tebuconazole no symptoms were observed, similar to water, DMSO, or 500$\mu \mathrm{M}$ CNI-1493 controls. In contrast, spikes sprayed with wild type and $500 \mu \mathrm{M}$ of CNI-1493, which is the maximum solubility of CNI-1493, showed a slight delay on infection symptoms in comparison with spikes infected with wild-type strain without the inhibitor (Supplementary Fig. S4). These results suggest efficiency of CNI-1493 inhibitor is less than the efficiency of a proper fungicide such as tebuconazole.

\section{Recombinant expression of DHS and in vitro inhibitor studies.}

To directly demonstrate that the DHS proteins of wheat and F. graminearum are targets of CNI-1493, the respective DHS cDNAs were expressed in Escherichia coli and were purified as histidine-tagged fusion proteins. Subsequently, these enzymes were analyzed with respect to their ability to incorporate radioactivity, which is transferred from $\left[{ }^{14} \mathrm{C}\right]$ spermidine to their homologous recombinant eIF-5A precursor protein (Sommer et al. 2004). To further control the assay system, we also included the human DHS in these analyses. As shown in Figure 9, all DHS enzymes were sensitive to CNI-1493 with respect to the modification of their homologous eIF-5A target protein. Clearly, Fusarium DHS, as compared with the human and wheat enzyme, was less active in this type of assay. Nonetheless, all enzymes displayed a dose-dependent inhibition profile, demonstrating that the guanylhydrazone CNI-1493 not only interferes as previously reported with human DHS (Hauber et al. 2005; Sommer et al. 2004) but also with the DHS enzymes from wheat and Fusarium.

\section{DISCUSSION}

The activity of eIF-5A protein can indirectly be monitored by inhibition of its activating enzyme DHS. The enzyme DHS performs the first of two spermidine-dependent reactions. These reactions modify a lysine of eIF-5A to hypusine, a highly conserved reaction in all eukaryotic cells (Park 2006; Wolff et al. 2007). In accordance to this, DHS and eIF-5A are also highly conserved proteins (Wolff et al. 2007).

Arabidopsis DHS transcription was induced under drought stress (Wang et al. 2003) and, in tomato, DHS has been increasingly expressed when senescence was induced by chilling stress (Wang et al. 2001). In our study, we were able to identify wheat
$D H S$ after inducing senescence. We show that wheat $D H S$ is induced and upregulated during pathogenic interactions, i.e., fungal infection, suggesting a role for $D H S$ expression in plant growth, stress, and defense.

Fusarium DHS proved also to be strongly induced during pathogenic interaction with its host plant, especially during the early stages of infection. Interestingly, this gene displayed different expressions on growth media. On rich medium, DHS is expressed in germinating conidia and rapidly dividing young mycelia. Transcription is clearly reduced after 6 days in older mycelium. In contrast, on poor medium the transcript is upregulated throughout from 3-day-old mycelia to 4-day and older mycelia. The high expression of fungal $D H S$ during plant infection and nutritional stress suggests a function of $D H S$ in pathogencity, germination, cell viability, and fungal proliferation.

To our knowledge, we investigated here, for the first time, the impact of CNI-1493 on the interaction of a pathogenic fungus with its host plants. We were able to demonstrate that, during infection of wheat spikes by $F$. graminearum, DHS transcription is induced in wheat as well as in the fungal cells. Interestingly, $D H S$ is not transcribed in wheat spikes during normal growth without stress. In fungal cells, however, the transcript of fungal DHS increased remarkably in infected spikes. We hypothesize that, in the host as well as in the pathogen, the protein eIF-5A transports, upon activation, mRNAs specifically needed during the pathogenic interaction.

The notion that eIF-5A participates in the posttranscriptional processing (i.e., stabilization, transport, and translation) of a subpopulation of specific mRNAs is supported by the finding that this protein exhibits RNA-binding properties (Xu and Chen 2001) and acts as a cellular cofactor of the HIV-1 Rev regulatory protein, which mediates the nucleocytoplasmic transport of specific retroviral transcripts (Bevec et al. 1996). In consequence, interference with the hypusine-modification of eIF-5A effectively inhibits the replication of HIV-1 or feline immunodeficiency virus, another Rev-encoding lentivirus (Andrus et al. 1998; Hart et al. 2002; Hauber et al. 2005; Schäfer et al. 2006). Thus, eIF-5A proved to selectively transport a subset of cellular mRNAs out of the nucleus to the ribosomes, thereby initiating their translation (Lee et al. 2009; Zanelli and Valentini. 2007). Furthermore, immunocytochemical analyses showed that the hypusinated eIF-5A is primarily localized in cytoplasm, while the nonhypusinated eIF-5A precursor distributes in both cytoplasm and nucleus (Lee et al. 2009).

The experimental DHS inhibitor CNI-1493 is under clinical investigation in various fields of medical importance, particularly in clinical trials for renal cancer, melanoma, or Crohn's disease (Sitaraman et al. 2003).

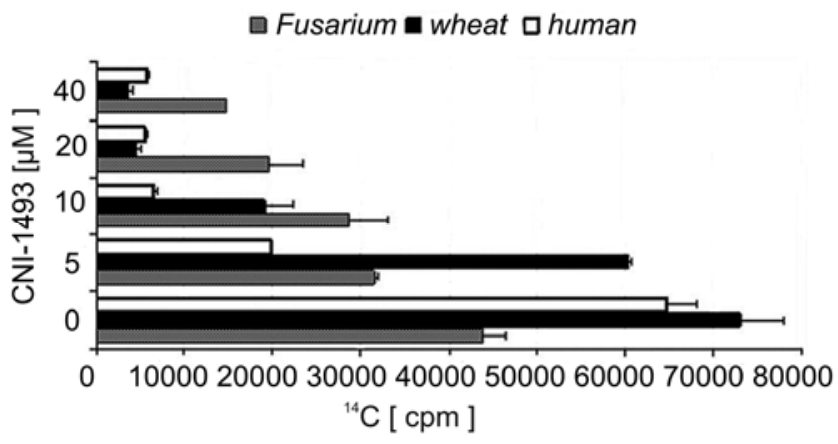

Fig. 9. Dose-dependent inhibition of deoxyhypusine synthase (DHS) activities by the guanylhydrazone CNI-1493. Recombinant Fusarium graminearum (gray bars), wheat (black bars), and human (white bars) DHS enzymes were analyzed in vitro to modify their respective homologous eIF-5A substrates in the presence of dimethyl sulfoxide (solvent control) or increasing concentrations of DHS inhibitor. 
The application of CNI-1493 in DHS enzyme assays in our study demonstrated that the guanylhydrazone CNI-1493 does not specifically interfere with human DHS but also with wheat and Fusarium DHS in a dose-dependent inhibition. We were able to prove in vitro that Fusarium and wheat DHS are targets of CNI-1493. The mechanisms by which CNI-1493 inhibit DHS are still unknown. Therefore, we can only speculate why Fusarium DHS is less inhibited compared with the human and wheat enzymes. F. graminearum DHS amino-acid sequence shows lower identity with wheat DHS and human DHS in comparison with the identity among them. Thus, Fusarium DHS could have less affinity to CNI-1493 than do wheat or human DHS in vitro, although DHS proteins are highly conserved at the active site (Umland et al. 2004).

Importantly, the application of CNI-1493 also resulted in a dose- and time-dependent inhibition of germination. A total block of germination was achieved at a concentration of 10 $\mu \mathrm{M}$ CNI-1493 at all studied timepoints. Germination of $F$. graminearum conidia was subdivided in four consecutive developmental steps, fresh conidia, activated conidia, germinated conidia, and developed hyphae. Fresh conidia are highly transcriptionally active; swollen and germinating conidia display a lower number of active genes, but a stage-specific gene expression occurs at all stages (Seong et al. 2008). Here, we studied the effect of CNI-1493 added at four different timepoints that are in accordance to these four developmental steps, fresh conidia, activated conidia, germinated conidia, and developed hyphae. CNI-1493 seems to already interfere at the early stages of conidia development (fresh conidia and conidia activation), because all conidia show a malformed appearance after 3 days of incubation, and no conidia were able to germinate properly and develop normal mycelia when CNI was added at $0 \mathrm{~h}$ (Fig. 4E). In addition, when $10 \mu \mathrm{M}$ or higher concentrations of CNI-1493 were added at later stages, all activated conidia, germinated conidia, or developed hyphae fail to continue growing. Seong and coworkers (2008) have shown that the transition from fresh to swollen conidia marks the largest change in gene expression. It is conceivable that CNI1493 interferes predominantly at this stage of conidia development, thus preventing the next developmental stage. However, late stages are also inhibited. More work is needed to investigate this inhibition on a molecular level.

In accordance with the inhibition of fungal germination, CNI-1493 displayed a powerful antifungal activity by controlling Fusarium infections of wheat and maize. When added to the fungal inoculum at a concentration of $10 \mu \mathrm{M}$, Fusarium infection was prevented completely and no visible symptoms were detectable. Surprisingly, the concomitant development of the plant kernel remained unaffected in spite of the application of the DHS inhibitor. The CNI-1493 treated wheat and maize plants did not show any negative effects, displaying normal growth and normal kernel development. Similarly, when wheat kernels were incubated in CNI-1493, even a concentration of $50 \mu \mathrm{M}$ affected neither kernel germination nor further seedling development.

The simultaneous inoculation of conidia and inhibitor in wheat spikelets lead to no symptoms at all (Fig. 6C), whereas infection is restricted to the inoculated spikelets when CNI1493 was added 3 hpi using the point-inoculation method (Fig. $8 B)$. Progression of infection is delayed using spray infection, suggesting the importance of the dose, time, and application method. Although the spray-inoculation method is a better approach to mimic a natural situation, point-inoculation experiments have the advantage of confining the sample at a specific point, with the inhibitor added at the same point and desired time. In addition, the progress of the infection can be followed through the inoculated spikelets.
In experiments carried out in vitro with tebuconazole in $F$. graminearum PH1 wild-type strain, Liu and associates (2010) reported that the fungal concentration that resulted in a $50 \%$ mycelial growth inhibition rate $\left(\mathrm{IC}_{50}\right)$ was $8 \mu \mathrm{M}(2.42 \mu \mathrm{g} / \mathrm{ml})$. The concentration of CNI-1493 necessary to suppress germination of $F$. graminearum in culture $(10 \mu \mathrm{M})$ is consistent with the $\mathrm{IC}_{50}$ of tebuconazole in vitro. However, the optimal concentration reported for tebuconazole to avoid infection with Fusarium species in the field are between 4 to $8 \mathrm{mM}$ (Paul et al 2007, 2008). Tebuconazole completely prevented Fusarium infection in our experiments, while CNI-1493 at a concentration of $500 \mu \mathrm{M}$ merely delayed the presence of symptoms. We suggest that this is due to the limited solubility of CNI-1493, which made it impossible to increase its concentration to the same level as tebuconazole.

At present, we can only speculate about the mechanism underlying the different susceptibility of wheat and Fusarium to CNI-1493. It is conceivable that the plant cuticle might prevent the uptake of the inhibitor. All aerial parts of plants are covered by a cuticle, whose major component is cutin, insoluble lipid-derived polyester. These plant cuticles function as permeability barriers for water and water-soluble materials, playing important roles in plant growth, environmental adaptation, and plant protection against environmental stresses (Kolattukudy 1996). In agreement with this hypothesis, it has previously been demonstrated that different cell lines take up DHS inhibitors differently and that their inhibitory effect varies in dependence on the uptake efficiency (Lee et al. 1995; Lee et al. 2003). This might reflect different inhibition levels of DHS enzyme activity in plants, such that complete suppression or deletion of the gene is lethal (Duguay et al. 2007). Therefore, the phenotype of a mutant plant with downregulated DHS expression depends on the remaining cellular amount of DHS (Thompson et al. 2004; Wang et al. 2001, 2003, 2005). In contrast, the selective suppression of $D H S$ in leaves enhanced growth without negative pleiotropic effects (Duguay et al. 2007).

A different explanation might be the observed induction of $D H S$ transcription during infection of wheat. $D H S$ transcripts of the invading fungus were already detectable 1 day after inoculation while, in contrast, the corresponding wheat gene transcripts were detectable only after 3 days. Keeping in mind that $D H S$ transcripts are not detectable in the spike during stress-free development, it might be reasonable that the early inhibition of fungal development by CNI-1493 prevents the induction of the wheat $D H S$ gene. Therefore, a DHS inhibitor is without effect in wheat because its DHS target is lacking.

Taken together, we characterized, for the first time, $D H S$ genes from wheat and $F$. graminearum and demonstrated their transcriptional induction during the pathogenic interaction. Furthermore, we demonstrated that the DHS inhibitor CNI1493 selectively inhibits fungal development and effectively prevents Fusarium infection of wheat and maize without disturbing plant development. Like eIF-5A, DHS genes are highly conserved (Wolff et al. 2007). Therefore, targeting fungal DHS by inhibitors appears to be a novel and valuable approach for protection of plant growth against fungal infection. Although, CNI-1493 is not applicable as a fungicide in the field, it might serve as a lead substance for the development of new antifungal agents.

\section{MATERIALS AND METHODS}

\section{Fungal and plant cultivation, conidia induction, and plant inoculation.}

F. graminearum 8/1, obtained from T. Miedaner (Miedaner et al. 2000) was cultured on the synthetic nutrient-poor medium SNA (Nirenberg 1981) and complete medium (Leach et 
al. 1982). Conidiation of $F$. graminearum was induced on SNA plates incubated at $18^{\circ} \mathrm{C}$ under near-UV white light as described (Jansen et al. 2005).

Wheat plants (Triticum aestivum L.) of the susceptible cultivars Florida or Nandu were grown in a greenhouse at 18 to $20^{\circ} \mathrm{C}, 60 \%$ humidity, and a photoperiod of $16 \mathrm{~h}$. Each of two central wheat spikelets was inoculated with $10 \mu \mathrm{l}$ of a suspension containing $2 \times 10^{4}$ conidia per milliliter (Jenczmionka et al. 2003). Maize plants (Zea maize L.) inbred line A188 were grown and were infected with $F$. graminearum conidia as described previously (Maier et al. 2006).

For spray-infection assays, $400 \mu \mathrm{l}$ of a conidial suspension with $1 \times 10^{5}$ conidia per milliliter of $F$. graminearum wild-type strain were sprayed on wheat spikes. CNI-1493 (600 $\mu \mathrm{l})$ at 200 or $500 \mu \mathrm{M}$ or $600 \mu \mathrm{l}$ of $2 \mathrm{mM}$ tebuconazole (Folicur 250, Bayer, Leverkusen, Germany) were sprayed 3 hpi. Controls used were $600 \mu \mathrm{l}$ of water, $10 \%$ DMSO, or $500 \mu \mathrm{M}$ CNI-1493. Percentage of spikelets infected divided by total number of spikelets per spike was used to analyze the severity of infection symptoms at different times after inoculation. Five spikes were used for each treatment. Each treatment was repeated at least three times.

\section{Inhibitory effect of CNI-1493}

on conidial germination in culture and kernels.

Examination of the inhibitory effect of CNI-1493 on conidial germination was performed in 96-well microtiter plates. To quantify mycelium growth with a fluorescence plate reader, a GFP-tagged $F$. graminearum wild type-like strain, WT- $g f p$, was used (Jansen et al. 2005), and $20 \mu \mathrm{l}$ of conidia suspension (concentration of $2 \times 10^{4}$ conidia/ml) and $180 \mu \mathrm{l}$ SNA medium were supplemented with $2 \mu \mathrm{l} \mathrm{CNI-1493}$ dissolved in DMSO, at indicated times and final concentrations. Pure DMSO $(2 \mu \mathrm{l})$ was used as a control. Microtiter plates were incubated at $28^{\circ} \mathrm{C}$ at $150 \mathrm{rpm}$. Precipitation occurred at concentrations greater than $500 \mu \mathrm{M}$ CNI-1493.

Inhibition studies of $F$. graminearum in culture were controlled microscopically. $F$. graminearum conidia $(n=1,000)$ were incubated in $200 \mu \mathrm{l}$ of SNA medium in single wells of a 96-well plate at $28^{\circ} \mathrm{C}$ and $150 \mathrm{rpm}$ shaking. CNI-1493 solutions $(2 \mu \mathrm{l})$ at concentrations of $10 \mathrm{nM}$ and $1 \mathrm{mM}$ were added to wells to determine inhibition potential of CNI-1493. As DMSO control, $2 \mu \mathrm{l}$ of DMSO were added to wells. Growth of four replicates of each concentration was studied. CNI-1493 was provided through the Department of Chemistry, Organic Chemistry, University of Hamburg.

To assess a possible influence of CNI-1493 on wheat kernel germination, wheat kernels were soaked in sterilized tap water with 0,10 , and $50 \mu \mathrm{M}$ CNI-1493 for $24 \mathrm{~h}$. Soaked kernels were put on water-soaked filter paper, were rolled up, and were put into a petri dish that contained water $(94 \mathrm{~mm})$. Germination and development of seedlings was assessed after 1 week.

\section{Molecular cloning of wheat $D H S$ cDNA.}

Sequence comparison of $D H S$ from A. thaliana and rice allowed the design of consensus primer pairs for reverse transcription (RT)-PCR (fwd: 5'-CCCTGGCCGCCGTNYTNAAR CA-3' and rev: 5'-CGAACCGGTCGATCATCTTGSWNGGN GTCCA-3'), (fwd: 5'-CGAGGTCGTCAACCAGATGHTNGA YTGG-3' and rev: 5'-TGTTGATGTACACGGCGTAGTCNGC NCCRTT-3'), (fwd: 5'-GGCTACCAGAAGACCACCATCTTY HTNGGNTA-3' and rev: 5'-GCCGGAGTCGGAGCCRTCRA AYTC-3'), (fwd: 5'-AACCTGCTGGTGCCGAAYGAYAAYT A-3' and rev: 5'-GCCCCAGGACACGGCYTCRTCNGG-3') and revealed a motif of $738 \mathrm{bp}$. Leaves of 4-week-old wheat plants were covered with aluminum foil for 4 days at $18^{\circ} \mathrm{C}$ and $60 \%$ humidity to induce $D H S$ transcription. RNA was isolated from leaves with the RNeasy plant mini kit (Qiagen, Hilden, Germany). The amplified motif was cloned into pGEM-T vector (Promega, Madison, WI, U.S.A.) and was sequenced to generate specific primers, to identify the complete sequence of DHS cDNA. The GeneRacer advanced race kit (Invitrogen, San Diego, CA, U.S.A.) was used to amplify the $5^{\prime}$ end of the gene with gene-specific primer (5'-CTGTATTTCGGGTCAAG TTCAGCTTC-3') and gene-nested specific primer (5'-CAGTC CTCGCTTGGCTTCTCA-3'), producing a fragment of 312 bp. The $3^{\prime}$ end of the gene was also amplified with genespecific primer (5'-CCATTCATGCAAGCCCAAGGAAGA-3') and nested gene-specific primer (5'-GGGTCATAATTCTTGG TGGAGGC- $3^{\prime}$ ), producing a fragment of 578 bp. Based on these two primers (fwd: 5'-GCGGAGGAGGCTCGTG-3' and rev: 5'-AAGTCACAGTTTTCCAGAGT-3') the full-length of DHS cDNA from wheat was amplified.

\section{Molecular cloning of $F$. graminearum DHS.}

A $F$. graminearum $D H S$ open reading frame was amplified from cDNA with the primers ( $f w d$ : $5^{\prime}$-GGCCTCCAACTCT GATGCTC-3' and rev: 5'-GATCCTCCTTCCCGATGTCC-3'), resulting in a fragment of $1,077 \mathrm{bp}$, which was cloned into pJET vector (Fermentas, St. Leon-Rot, Germany) and was sequenced. DHS genes from wheat and $F$. graminearum were amplified with an initial denaturation at $94^{\circ} \mathrm{C}$ for $3 \mathrm{~min}, 35 \mathrm{cy}-$ cles of $94^{\circ} \mathrm{C}$ for $30 \mathrm{~s}$, annealing for $45 \mathrm{~s}$ at 55 to $59^{\circ} \mathrm{C}$, and extension for $2 \mathrm{~min}$ at $72^{\circ} \mathrm{C}$. The final extension step was performed at $72^{\circ} \mathrm{C}$ for $5 \mathrm{~min}$.

\section{Expression analysis by RT-PCR.}

Germination of conidia for expression analysis was performed according to Jenczmionka and coworkers (2003). To study the expression patterns of the $D H S$ gene in wheat and F. graminearum, tissues were frozen in liquid nitrogen before RNA isolation. The RNeasy plant and fungi kit (Qiagen) and the RevertAid $\mathrm{H}$ minus first-strand cDNA synthesis kit (Fermentas) were used, according to the manufacturer's instructions.

In wheat, PCR reactions were carried out using the primer pairs fwd: 5'-GCGGAGGAGGCTCGTG-3' and rev: 5'-AAGT CACAGTTTTCCAGAGT-3', while the 18S-rRNA intron spanning primers $f w d$ : 5'-CTGCCAGTAGTCATATGCTTGTCT-3' and rev: 5'-CCCCGTGTCAGGATTGG-3' were used as expression control of cDNA. In $F$. graminearum, PCR reactions were carried out with the specific primers fwd: $5^{\prime}$-GGCCT CCAACTCTGATGCTC-3' and rev: 5'-GATCCTCCTTCCC GATGTCC-3'. $\beta$-tubulin mRNA served as reference with the intron spanning primers fwd: $5^{\prime}$-TGCTGTTCTGGTCGATCT TG-3' and rev: 5'-ATGAAGAAGTGAAGTCGGGG-3'). Reaction conditions in the reverse transcription step for the expression analysis were based on the use of the housekeeping gene $\beta$-tubulin in F. graminearum and $18 S$-rRNA in wheat, as internal controls. Semiquantitative RT-PCR was conducted with 25 or 28 PCR cycles.

Expression and purification of recombinant fusion proteins.

The plasmid pGEX-eIF-5A, which has been previously described (Bevec et al. 1996), is a bacterial expression vector in which the human $e I F-5 A$ cDNA is fused in-frame to the $3^{\prime}$ end of the glutathione $S$-transferase (GST) coding region of the vector pGEX-3X (Amersham Biosciences, Piscataway, NJ, U.S.A.). Likewise, the eIF-5A cDNA from wheat and $F$. graminearum was inserted between the Bam $\mathrm{HI}$ and EcoRI site of pGEX-3X. For bacterial expression of His-tagged DHS fusion protein, the cDNAs of human, wheat, and $F$. graminearum DHS were inserted between the BamHI and EcoRI sites of pTrcHis-C (Invitrogen). N-terminally His-tagged human, wheat, and Fusarium DHS or the various GST-eIF-5A fusion proteins 
were expressed in E. coli DH-5 $\alpha$ (BL21-DE3), using the respective pTrc-DHS or pGEX-eIF-5A vector. Protein purification and removal of the GST moiety from the GST-eIF-5A fusion by factor Xa cleavage was performed as described in detail earlier (Sommer et al. 2004).

\section{DHS assay.}

DHS activity was measured by the incorporation of radioactivity derived from $\left[{ }^{14} \mathrm{C}\right]$ spermidine into recombinant eIF-5A precursor protein. The reaction mixture contained $5 \mu \mathrm{g}$ of eIF$5 \mathrm{~A}, 0.25 \mu \mathrm{Ci}\left[{ }^{14} \mathrm{C}\right]$ spermidine trihydrochloride $(50 \mu \mathrm{Ci} / \mathrm{ml}), 1$ $\mathrm{mM}$ of NAD+, $1 \mathrm{mM}$ of dithiothreitol, $50 \mu \mathrm{g}$ of bovine serum albumin per milliliter, and $2 \mu \mathrm{g}$ of purified DHS enzyme in 0.3 $\mathrm{M}$ glycine- $\mathrm{NaOH}$ buffer, $\mathrm{pH} 9.0$ (total volume $200 \mu \mathrm{l}$ ). After 2 $\mathrm{h}$ of incubation at $37^{\circ} \mathrm{C}$, the reaction was terminated by the addition of $100 \mu \mathrm{l}$ of $20 \mathrm{mM}$ spermidine, and the mixture was adsorbed to a GSWP02500 nitrocellulose filter (Millipore, Bedford, MA, U.S.A.). Subsequently, the filter was washed with $2.5 \mathrm{ml}$ of phosphate buffered saline and was dried at ambient temperature. Radioactivity was measured with a liquid scintillation spectrometer.

\section{ACKNOWLEDGMENTS}

We thank B. Doormann and J. Bormann for critically reading the manuscript.

\section{LITERATURE CITED}

Andrus, L., Szabo, P., Grady, R. W., Hanauske, A. R., Huima-Byron, T. Slowinska, B., Zagulska, S. and Hanauske-Abel, H. M. 1998. Antiretroviral effects of deoxyhypusyl hydroxylase inhibitors: A hypusine-dependent host cell mechanism for replication of human immunodeficiency virus type 1 (HIV-1). Biochem. Pharmacol. 55:1807-1818.

Bevec, D., Jaksche, H., Oft, M., Wohl, T., Himmelspach, M., Pacher, A. Schebesta, M., Koettnitz, K., Dobrovnik, M., Csonga, R., Lottspeich, F. and Hauber, J. 1996. Inhibition of HIV-1 replication in lymphocytes by mutants of the Rev cofactor eIF-5A. Science 271:1858-1860.

Cano, V. S., Jeon, G. A., Johansson, H. E., Henderson, C. A., Park, J. H., Valentini, S. R., Hershey, J. W. and Park, M. H. 2008. Mutational analyses of human eIF5A-1-identification of amino acid residues critical for eIF5A activity and hypusine modification. FEBS (Fed. Eur. Biochem. Soc.) J. 275:44-58.

Dias, C. A., Cano, V. S., Rangel, S. M., Apponi, L. H., Frigieri, M. C., Muniz, J. R., Garcia, W., Park, M. H., Garratt, R. C., Zanelli, C. F. and Valentini, S. R. 2008. Structural modeling and mutational analysis of yeast eukaryotic translation initiation factor $5 \mathrm{~A}$ reveal new critical residues and reinforce its involvement in protein synthesis. FEBS (Fed. Eur. Biochem. Soc.) J. 275:1874-1888.

Duguay, J., Jamal, S., Liu, Z., Wang, T. W. and Thompson, J. E. 2007. Leaf-specific suppression of deoxyhypusine synthase in Arabidopsis thaliana enhances growth without negative pleiotropic effects. J. Plant Physiol. 164:408-420.

Foster, S. A. and Walters, D. R. 1993. Fungicidal Activity of the Polyamine Analog, Keto-Putrescine. Pestic. Sci. 37:267-272.

Frigieri, M. C., Joao Luiz, M. V., Apponi, L. H., Zanelli, C. F. and Valentini, S. R. 2008. Synthetic lethality between eIF5A and Ypt1 reveals a connection between translation and the secretory pathway in yeast. $\mathrm{Mol}$ Genet Genomics. 280:211-221.

Hanauske-Abel, H. M., Slowinska, B., Zagulska, S., Wilson, R. C., Staiano-Coico, L., Hanauske, A. R., McCaffrey, T. and Szabo, P. 1995. Detection of a sub-set of polysomal mRNAs associated with modulation of hypusine formation at the G1-S boundary. Proposal of a role for eIF-5A in onset of DNA replication. FEBS (Fed. Eur. Biochem. Soc.) Lett. 366:92-98.

Hart, R. A., Billaud, J. N., Choi, S. J. and Phillips, T. R. 2002. Effects of 1,8-diaminooctane on the FIV Rev regulatory system. Virology. 304:97104

Hauber, I., Bevec, D., Heukeshoven, J., Kratzer, F., Horn, F., Choidas, A., Harrer, T. and Hauber, J. 2005. Identification of cellular deoxyhypusine synthase as a novel target for antiretroviral therapy. J Clin Invest. 115:76-85.

Havis, N. D., Walters, D. R., Foster, S. A., Martin, W. P., Cook, F. M. and Robins, D. J. 1994. Fungicidal activity of the synthetic putrescine analog, (E)-1,4-Diaminobut-2-Ene, and Derivatives. Pesticide Science. 41:61-69.
Homdork, S., Fehrmenn, H., Beck, R., 2000. Effects of field application of tebuconazole on yield, yield components and the mycotoxin content of Fusarium-infected wheat grain. J. Phytopathol. 148:1-6.

Jakus, J., Wolff, E. C., Park, M. H. and Folk, J. E. 1993. Features of the spermidine-binding site of deoxyhypusine synthase as derived from inhibition studies. Effective inhibition by bis- and mono-guanylated diamines and polyamines. J. Biol. Chem. 268:13151-13159.

Jansen, C., von Wettstein, D., Schafer, W., Kogel, K. H., Felk, A. and Maier, F. J. 2005. Infection patterns in barley and wheat spikes inoculated with wild-type and trichodiene synthase gene disrupted Fusarium graminearum. Proc. Natl. Acad. Sci. U.S.A. 102:16892-16897.

Jenczmionka, N. J., Maier, F. J., Lösch, A. P. and Schäfer, W. 2003. Mating, conidiation and pathogenicity of Fusarium graminearum, the main causal agent of the head-blight disease of wheat, are regulated by the MAP kinase gpmk1. Curr. Genet. 43:87-95.

Kolattukudy, P. E. 1996. Biosynthetic pathways of cutin and waxes, and their sensitivity to environmental stresses. In Plant Cuticles. Edited by Kerstiens, G. BIOS Scientific publishers Ltd., Oxford. pp 83-108.

Leach, J., Lang, B. R. a. and Yoder, O. C. 1982. Methods for selection of mutants and in vitro culture of Cochliobolus heterostrophus. J. Gen. Microbiol. 128:1719-1729.

Lee, J., Lee, T., Lee, Y. W., Yun, S. H. and Turgeon, B. G. 2003. Shifting fungal reproductive mode by manipulation of mating type genes: Obligatory heterothallism of Gibberella zeae. Mol. Microbiol. 50:145152.

Lee, S. B., Park, J. H., Kaevel, J., Sramkova, M., Weigert, R. and Park, M. H. 2009. The effect of hypusine modification on the intracellular localization of eIF5A. Biochem Biophys Res Commun. 383:497-502.

Lee, Y. B., Park, M. H. and Folk, J. E. 1995. Diamine and triamine analogs and derivatives as inhibitors of deoxyhypusine synthase: Synthesis and biological activity. J. Med. Chem. 38:3053-3061.

Liu, X., Jiang, J., Shao, J., Yin, Y. and Ma, Z. 2010. Gene transcription profiling of Fusarium graminearum treated with an azole fungicide tebuconazole. Appl. Microbiol. Biotechnol. 85:1105-1114.

Mackintosh, C. A. and Walters, D. R. 1997a. Fungicidal activity of two spermidine analogues. J Phytopathol. 145:43-45.

Mackintosh, C. A. and Walters, D. R. 1997b. Growth and polyamine metabolism in Pyrenophora avenae exposed to cyclohexylamine and norspermidine. Amino Acids 13:347-354.

Mackintosh, C. A. and Walters, D. R. 2003. Antifungal activity of some deoxyhypusine synthase inhibitors (short communication: Plant mycology and crop protection). Mycopathologia 156:329-332.

Maier, F. J., Miedaner, T., Hadeler, B., Felk, A., Salomon, S., Lemmens, M., Kassner, H. and Schäefer, W. 2006. Involvement of trichothecenes in fusarioses of wheat, barley, and maize evaluated by gene disruption of the trichodiene synthase (Tri5) gene in three field isolates of different chemotype and aggressiveness. Mol. Plant Pathol. 6:449-461.

Miedaner, T., Reinbrecht, C. and Schilling, A. G. 2000. Association among aggressiveness, fungal colonization, and mycotoxin production of 26 isolates of Fusarium graminearum in winter rye head blight. J. Plant Dis. Prot. 107:124-134.

Nirenberg, H. I. 1981. A simplified method for identifying Fusarium Spp occurring on Wheat. Canadian J. Bot. 59:1599-1609.

Park, M. H. 2006. The post-translational synthesis of a polyamine-derived amino acid, hypusine, in the eukaryotic translation initiation factor 5A (eIF5A). J. Biochem. 139:161-169.

Park, M. H., Wolff, E. C. and Folk, J. E. 1993a. Hypusine: Its post-translational formation in eukaryotic initiation factor $5 \mathrm{~A}$ and its potential role in cellular regulation. Biofactors 4:95-104.

Park, M. H., Wolff, E. C. and Folk, J. E. 1993b. Is hypusine essential for eukaryotic cell proliferation? Trends Biochem. Sci. 18:475-479.

Park, M. H., Wolff, E. C., Lee, Y. B. and Folk, J. E. 1994. Antiproliferative effects of inhibitors of deoxyhypusine synthase. Inhibition of growth of Chinese hamster ovary cells by guanyl diamines. J. Biol. Chem 269:27827-27832.

Paul, P. A., Lipps, P. E., Hershman, D. E., McMullen, M. P., Draper, M. A., and Madden, L. V. 2007. A quantitative review of tebuconazole effect on Fusarium Head Blight and deoxynivalenol content in wheat. Phytopathology 97:211-220.

Paul, P. A., Lipps, P. E., Hershman, D. E., McMullen, M. P., Draper, M. A, and Madden, L. V. 2008. Efficacy of triazole-based fungicides for Fusarium head blight and deoxynivalenol control in wheat: A multivariate meta-analysis. Phytopathology 98:999-1011.

Ruhl, M., Himmelspach, M., Bahr, G. M., Hammerschmid, F., Jaksche, H., Wolff, B., Aschauer, H., Farrington, G. K., Probst, H. and Bevec, D. 1993. Eukaryotic initiation factor $5 \mathrm{~A}$ is a cellular target of the human immunodeficiency virus type 1 Rev activation domain mediating transactivation. J. Cell Biol. 123:1309-1320.

Sasaki, K., Abid, M. R. and Miyazaki, M. 1996. Deoxyhypusine synthase gene is essential for cell viability in the yeast Saccharomyces cere 
visiae. FEBS (Fed. Eur. Biochem. Soc.) Lett. 384:151-154

Schäfer, B., Hauber, I., Bunk, A., Heukeshoven, J., Dusedau, A., Bevec, D. and Hauber, J. 2006. Inhibition of multidrug-resistant HIV-1 by interference with cellular S-adenosylmethionine decarboxylase activity. J. Infect. Dis. 194:740-750.

Schnier, J., Schwelberger, H. G., Smit-McBride, Z., Kang, H. A. and Hershey, J. W. 1991. Translation initiation factor 5A and its hypusine modification are essential for cell viability in the yeast Saccharomyces cerevisiae. Mol. Cell Biol. 11:3105-3114.

Seong, K. Y., Zhao, X., Xu, J. R., Guldener, U. and Kistler, H. C. 2008. Conidial germination in the filamentous fungus Fusarium graminearum. Fungal Genet. Biol. 45:389-399.

Sitaraman, S. V., Hoteit, M. and Gewirtz, A. T. 2003. Semapimod. Cytokine. Curr. Opin. Investig. D. 4:1363-1368.

Sommer, M. N., Bevec, D., Klebl, B., Flicke, B., Holscher, K., Freudenreich, T., Hauber, I., Hauber, J. and Mett, H. 2004. Screening assay for the identification of deoxyhypusine synthase inhibitors. J. Biomol. Screen. 9:434-438.

Specht, S., Sarite, S. R., Hauber, I., Hauber, J., Gorbig, U. F., Meier, C., Bevec, D., Hoerauf, A. and Kaiser, A. 2008. The guanylhydrazone CNI1493: An inhibitor with dual activity against malaria-inhibition of host cell pro-inflammatory cytokine release and parasitic deoxyhypusine synthase. Parasitol. Res. 102:1177-1184.

Stevens, L. and Winther, M. D. 1979. Spermine, spermidine and putrescine in fungal development. Adv. Microb. Physiol. 19:63-148.

Thompson, J. D., Higgins, D. G. and Gibson, T. J. 1994. CLUSTAL W: Improving the sensitivity of progressive multiple sequence alignment through sequence weighting, position-specific gap penalties and weight matrix choice. Nucleic Acids Res. 22:4673-4680.

Thompson, J. E., Hopkins, M. T., Taylor, C. and Wang, T. W. 2004. Regu- lation of senescence by eukaryotic translation initiation factor 5A: Implications for plant growth and development. Trends Plant Sci. 9:174179.

Umland, T. C., Wolff, E. C., Park, M. H. and Davies, D. R. 2004. A new crystal structure of deoxyhypusine synthase reveals the configuration of the active enzyme and of the xNADxinhibitor ternary complex. J. Biol. Chem 27:28697-28705.

Wang, T. W., Lu, L., Wang, D. and Thompson, J. E. 2001. Isolation and characterization of senescence-induced cDNAs encoding deoxyhypusine synthase and eukaryotic translation initiation factor 5A from tomato. J. Biol. Chem. 276:17541-17549.

Wang, T. W., Lu, L., Zhang, C. G., Taylor, C. and Thompson, J. E. 2003. Pleiotropic effects of suppressing deoxyhypusine synthase expression in Arabidopsis thaliana. Plant Mol. Biol. 52:1223-1235.

Wang, T. W., Zhang, C. G., Wu, W., Nowack, L. M., Madey, E. and Thompson, J. E. 2005. Antisense suppression of deoxyhypusine synthase in tomato delays fruit softening and alters growth and development. Plant Physiol. 138:1372-1382.

Wolff, E. C. and Park, M. H. 1999. Identification of lysine350 of yeast deoxyhypusine synthase as the site of enzyme intermediate formation. Yeast 15:43-50.

Wolff, E. C., Kang, K. R., Kim, Y. S. and Park, M. H. 2007. Posttranslational synthesis of hypusine: Evolutionary progression and specificity of the hypusine modification. Amino Acids. 33:341-350.

$\mathrm{Xu}, \mathrm{A}$. and Chen, K. Y. 2001. Hypusine is required for a sequence-specific interaction of eukaryotic initiation factor $5 \mathrm{~A}$ with postsystematic evolution of ligands by exponential enrichment RNA. J. Biol. Chem. 276:2555-2561.

Zanelli, C. F. and Valentini, S. R. 2007. Is there a role for eIF5A in translation? Amino Acids 33:351-358. 